\title{
Ex Vivo and In Vivo Evaluation of Overexpressed VLA-4 in Multiple Myeloma Using LLP2A Imaging Agents
}

\author{
Deepti Soodgupta*1, Haiying Zhou*2, Wissam Beaino ${ }^{3}$, Lan Lu${ }^{1}$, Michael Rettig ${ }^{1}$, Mark Snee ${ }^{4}$, James Skeath ${ }^{4}$, \\ John F. DiPersio ${ }^{1}$, Walter J. Akers ${ }^{2}$, Richard Laforest ${ }^{2}$, Carolyn J. Anderson ${ }^{3}$, Michael H. Tomasson ${ }^{1}$, \\ and Monica Shokeen ${ }^{2}$ \\ ${ }^{1}$ Division of Oncology, Department of Medicine, Washington University Medical School, St. Louis, Missouri; ${ }^{2}$ Mallinckrodt Institute \\ of Radiology, Washington University Medical School, St. Louis, Missouri; ${ }^{3}$ Department of Radiology, University of Pittsburgh, \\ Pittsburgh, Pennsylvania; and ${ }^{4}$ Department of Genetics, Washington University Medical School, St. Louis, Missouri
}

\begin{abstract}
Very-late-antigen-4 (VLA-4, $a_{4} \beta_{1}$ integrin, CD49d/CD29) is a transmembrane adhesion receptor that plays an important role in cancer and immune responses. Enhanced VLA-4 expression has been observed in multiple myeloma (MM) cells and surrounding stroma. VLA-4 conformational activation has been associated with MM pathogenesis. VLA-4 is a promising MM imaging and therapeutic biomarker. Methods: Specificity of ${ }^{64} \mathrm{CU}$-LLP2A $\left({ }^{64} \mathrm{Cu}-\mathrm{CB}-\mathrm{TE} 1 \mathrm{~A} 1 \mathrm{P}-\right.$ $\mathrm{PEG}_{4}$-LLP2A), a high-affinity VLA-4 peptidomimetic-based radiopharmaceutical, was evaluated in $a_{4}$ knock-out mice and by competitive blocking in wild-type tumor-bearing mice. ${ }^{64} \mathrm{Cu}$-LLP2A PET/CT (static and dynamic) imaging was conducted in C57BL6/KaLwRij mice bearing murine 5TGM1-GFP syngeneic tumors generated after intravenous injection via the tail. Blood samples were collected for serum protein electrophoresis. Bone marrow and splenic cells extracted from tumor-bearing and control mice ( $n=3$ /group) were coincubated with the optical analog LLP2A-Cy5 and mouse B220, CD4, Gr1, and Mac1 antibodies and analyzed by fluorescenceactivated cell sorting. Human radiation dose estimates for ${ }^{64} \mathrm{Cu}-\mathrm{LLP} 2 \mathrm{~A}$ were extrapolated from mouse biodistribution data (6 time points, $0.78 \mathrm{MBq}$ /animal, $n=4 /$ group). Ten formalin-fixed paraffinembedded bone marrow samples from deceased MM patients were stained with LLP2A-Cy5. Results: ${ }^{64} \mathrm{Cu}-\mathrm{LLP} 2 \mathrm{~A}$ and LLP2A-Cy5 demonstrated high specificity for VLA-4-positive mouse 5TGM1-GFP myeloma and nonmalignant inflammatory host cells such as $T$ cells and $\mathrm{myeloid} / \mathrm{monocytic}$ cells. Ex vivo flow cytometric analysis supported a direct effect of myeloma on increased VLA-4 expression in host hematopoietic microenvironmental elements. SUVs and the number of medullar lesions detected by ${ }^{64} \mathrm{Cu}$-LLP2A PET corresponded with increased monoclonal $(\mathrm{M})$ protein $(\mathrm{g} / \mathrm{dL})$ in tumor-bearing mice over time $(3.29 \pm 0.58$ at week 0 and $9.97 \pm 1.52$ at week 3$)$. Dynamic PET with ${ }^{64} \mathrm{Cu}$-LLP2A and ${ }^{18} \mathrm{~F}-\mathrm{FDG}$ demonstrated comparable SUV in the prominent lesions in the femur. Human radiation dose estimates indicated urinary bladder wall as the dose-limiting organ $(0.200 \mathrm{mGy} / \mathrm{MBq})$, whereas the dose to the red marrow was $0.006 \mathrm{mGy} / \mathrm{MBq}$. The effective dose was estimated to be $0.017 \mathrm{mSv} / \mathrm{MBq}$. Seven of the ten human samples displayed a high
\end{abstract}

Received Aug. 6, 2015; revision accepted Nov. 25, 2015. For correspondence or reprints contact either of the following:

Monica Shokeen, Department of Radiology, Mallinckrodt Institute of

Radiology, 4515 McKinley Avenue, 2nd floor, St. Louis, MO 63110.

E-mail: mshokeen@wustl.edu

Michael H. Tomasson, Division of Oncology, Department of Medicine, Washington University School of Medicine, 660 S. Euclid Ave., St. Louis, MO 63110.

E-mail: tomasson@dom.wustl.edu

${ }^{*}$ Contributed equally to this work.

Published online Jan. 7, 2016.

COPYRIGHT (C) 2016 by the Society of Nuclear Medicine and Molecular Imaging, Inc. proportion of cells intensely labeled with LLP2A-Cy5 probe. Conclusion: ${ }^{64}$ Cu-LLP2A and LLP2A-Cy5 demonstrated binding specificity for VLA-4 in an immune-competent murine MM model. ${ }^{64} \mathrm{Cu}-\mathrm{LLP} 2 \mathrm{~A}$ displayed favorable dosimetry for human studies and is a potential imaging candidate for overexpressed VLA-4.

Key Words: animal imaging; molecular imaging; radiobiology/dosimetry; dosimetry; LLP2A; multiple myeloma; VLA-4 specificity

J Nucl Med 2016; 57:640-645

DOI: 10.2967/jnumed.115.164624

$\mathbf{M}$ ultiple myeloma (MM) is the second most common hematologic cancer in the United States, diagnosed in approximately 25,000 Americans each year, and is currently incurable in most patients (1). MM is an age-related malignancy of plasma B cells, characterized by hallmark genetic mutations and changes in the hematopoietic microenvironment (2). The end-organ damage caused by MM includes lytic bone lesions, anemia, immunodeficiency, hypercalcemia, and renal insufficiency. Myeloma cells tend to resculpt the bone microenvironment by facilitating neoangiogenesis, recruiting tumor-associated macrophages, suppressing osteoblasts, and stimulating osteoclasts via soluble activating factors. $\mathrm{MM}$ and its obligate precursor state, monoclonal gammopathy of uncertain significance, are readily detected using serum markers (either intact immunoglobulin or free light chains) in $90 \%$ of cases. However, serum markers are insufficient to distinguish premalignant monoclonal gammopathy of uncertain significance and smoldering MM from fully transformed MM. The diagnosis of MM requires very high monoclonal tumor burden or end-organ damage, such as lytic bone lesions, and highly sensitive radiographic skeletal imaging, which is an integral part of the diagnosis of MM (3). Evaluation of progression and treatment response is also confounded in $10 \%$ of $\mathrm{MM}$ patients who display an oligosecretory phenotype (defined as serum M-protein $<1 \mathrm{~g} / \mathrm{dL}$ and urine M-protein $<200 \mathrm{mg} / 24 \mathrm{~h}$ ) (4). The timely and accurate diagnosis of MM is important because a delay in the diagnosis of MM can be detrimental to the patient's outcome (3).

Whole-body imaging approaches such as noninvasive molecularly targeted PET can sensitively, quantitatively, and spatiotemporally probe functional biologic processes such as changes in receptor expression during disease progression. Most clinical PET imaging in $\mathrm{MM}$ is performed with the metabolic tracer ${ }^{18} \mathrm{~F}$-labeled FDG ( $\left.{ }^{18} \mathrm{~F}-\mathrm{FDG}\right)$; however, MM cells are hypoproliferative and do not 
consistently overexpress glucose transporter 1 , and over a third of intramedullary myeloma lesions can go undetected by ${ }^{18} \mathrm{~F}$-FDG PET (5).

Very-late-antigen-4 (VLA-4, $\alpha_{4} \beta_{1}$ receptor, CD49d/CD29) is a transmembrane adhesion receptor that plays an important role in normal immune responses as well as cancer pathogenesis (6). Enhanced VLA-4 expression has been observed in MM cells and surrounding stroma. Additionally, myeloma bone marrow stroma is rich in the VLA-4 ligands, vascular cell adhesion molecule-1, and fibronectin (7). In MM, inside-out signaling induces a VLA-4 conformational change to an activated state triggering high-affinity ligand binding, which in turn activates further intracellular signaling (outsidein signaling) (8). VLA-4 conformational activation is correlated with enhanced cell trafficking, proliferation, and drug resistance (9).

LLP2A is a high-affinity peptidomimetic ligand for VLA-4, and bioconjugates of LLP2A have shown promise as imaging and therapeutic agents (10-12). We previously demonstrated the proofof-principle molecular imaging of VLA-4 in murine 5TGM1 myeloma subcutaneous tumors using the PET radiopharmaceutical ${ }^{64} \mathrm{Cu}$-(CB-TE1A1P)-LLP2A (12). Recently Beaino et al. demonstrated imaging of VLA-4-positive melanoma mouse models using ${ }^{64} \mathrm{Cu}-\mathrm{CB}-\mathrm{TE} 1 \mathrm{~A} 1 \mathrm{P}-\mathrm{PEG}_{4}$-LLP2A $\left({ }^{64} \mathrm{Cu}-\mathrm{LLP} 2 \mathrm{~A}\right)(13) .{ }^{64} \mathrm{Cu}-\mathrm{LLP} 2 \mathrm{~A}$ possesses ideal pharmacokinetic features such as optimal bioavailability (by virtue of added PEG chains), enhanced solubility, and high ${ }^{64} \mathrm{Cu}$ specific activity $(\sim 37 \mathrm{MBq} / \mu \mathrm{g})$.

In the current study, we evaluated the specificity of nuclear and optical analogs of LLP2A for imaging overexpressed VLA-4 in the hematopoietic environments in MM using intravenous syngeneic MM mouse models, estimated radiation dose-limiting organs through normal mouse biodistribution, and stained human tissues for VLA-4 positivity. We additionally evaluated the specificity of LLP2A for VLA-4-positive murine 5TGM1-GFP myeloma and nonmalignant host inflammatory stromal cells including $\mathrm{T}$ and $\mathrm{B}$ lymphocytes and macrophages in vivo and ex vivo. Longitudinal ${ }^{64} \mathrm{Cu}-\mathrm{LLP} 2 \mathrm{~A}$ PET was performed in a syngeneic C57BL6/KaLwRij/5TGM1-GFP mouse model of systemic (tumor cells injected intravenously via the tail) MM to monitor medullar tumor lesions over the disease progression. Binding specificity of LLP2A to tumor cells and tumor-associated inflammatory cells in the bone marrow and spleen were quantified by flow cytometry using fluorescent-labeled LLP2A (LLP2A-Cy5). VLA-4-targeted PET imaging with ${ }^{64} \mathrm{Cu}$-LLP2A was compared with ${ }^{18} \mathrm{~F}-\mathrm{FDG} \mathrm{PET}$, and human radiation dose estimates for ${ }^{64} \mathrm{Cu}-\mathrm{LLP} 2 \mathrm{~A}$ were extrapolated from mouse biodistribution data. Finally, to determine whether LLP2A can be used to follow VLA-4 activation/overexpression in human MM tissue, we stained 10 formalin-fixed paraffin-embedded bone marrow samples from deceased MM patients with LLP2A-Cy5.

\section{MATERIALS AND METHODS}

\section{Synthesis and Radiolabeling of ${ }^{64} \mathrm{Cu}$-LLP2A}

CB-TE1A1P-PEG 4 -LLP2A was synthesized and radiolabeled as described previously (13). The supplemental data provide details (supplemental materials are available at http://jnm.snmjournals.org).

\section{Synthesis of LLP2A-Cy5}

Sulfo-cyanine5 NHS ester (Lumiprobe; a hydrophilic dye), with 646/ 662 peak excitation/emission in dimethyl sulfoxide, was conjugated to LLP2A. The supplemental data provide details (Supplemental Figs. 1-3).

\section{Flow Cytometry}

MM Cell Lines. Cells were grown and analyzed by fluorescenceactivated cell sorting as previously discussed (14). The supplemental data provide details.
Bone Marrow and Spleen Tissues. All animal studies were conducted under a protocol approved by the Washington University Institutional Animal Care and Use Committee. The supplemental data provide details on tissue processing for fluorescence-activated cell sorting.

\section{$\boldsymbol{a}_{4}-/-$ Mice}

The $\alpha_{4}-/-$ mice were a generous gift from Dr. Thalia Papayannopoulou, University of Washington School of Medicine. These mice express Tek cre(tie 2 cre), which deletes the VLA-4 in all endothelial and hematopoietic cells (germline deletion) $(15,16)$.

\section{Serum Protein Electrophoresis}

Serum protein electrophoresis was performed as described previously (12). The supplemental data provide details.

\section{Small-Animal PET/CT Imaging}

Small-animal PET/CT imaging was performed as described previously (12). The supplemental data provide details.

\section{Human Radiation Dosimetry}

The human radiation doses of ${ }^{64} \mathrm{Cu}$-LLP2A were estimated for the adult anthropomorphic male model using biodistribution data in BALB/c (albino laboratory-bred strain; The Jackson Laboratory) mice. Five groups of BALB/c mice $(n=5)$ were injected with $0.78 \mathrm{MBq}$ of the tracer, and organs were collected at $1,2,4,12,24$, and $48 \mathrm{~h}$ after injection. The percentage injected dose per gram of tissue was determined by decay correction of the radiopharmaceutical for each sample. The last group of animals was maintained in metabolic cages to collect excreted urine and feces. Time-activity curves were created from the collected data expressed in percentage of injected dose/activity. The supplemental data provide details on human radiation dosimetry calculations.

\section{Staining of Primary Human Tissues with LLP2A-Cy5}

Studies with human samples were approved by the human research protection office at Washington University in St. Louis, and informed consent from the patients was obtained in accordance with the Declaration of Helsinki. Animal studies were approved by the Animal Study Committee at Washington University in St. Louis. The supplemental data provide details on the staining protocol.

\section{Data Analysis and Statistics}

All data are presented as mean $\pm \mathrm{SD}$. Groups were compared using PRISM 5.0 (GraphPad) 2-tailed Student $t$ test. $P$ values of less than 0.05 were considered statistically significant.

\section{RESULTS}

\section{In Vivo Binding Specificity of ${ }^{64} \mathrm{Cu}-$ LLP2A to 5TGM1-GFP Murine MM Cells}

The ${ }^{64} \mathrm{Cu}$-LLP2A used in this study is the modified ( $\mathrm{PEG}_{4}$ added) version of the original analog (12). In the subcutaneous 5TGM1-GFP murine myeloma model, intravenously injected ${ }^{64} \mathrm{Cu}$-LLP2A demonstrated excellent tumor uptake. The radiopharmaceutical was taken up and selectively retained by the myeloma tumor cells as demonstrated in the 24-h postinjection mouse PET image and the autoradiographic slice from the excised tumor (Fig. 1A). The uptake of the radiopharmaceutical was reduced to the background levels when coinjected with a 200-fold excess of cold-LLP2A (Fig. 1A, right). As compared with age-matched wild-type KaLwRij mice, tumor-free $\alpha_{4}-/-$ mice showed negligible uptake in the spleen (Fig. 1B), indicating that LLP2A uptake in the spleens of normal mice is VLA-4-mediated.

\section{Small-Animal PET Imaging with ${ }^{64} \mathrm{Cu}-\mathrm{LLP2A}$ in Disseminated Murine Myeloma Model}

In the 5TGM1-GFP metastatic/intravenous MM mouse model, the tumor cells tend to engraft in the hematopoietic organs, primarily the 


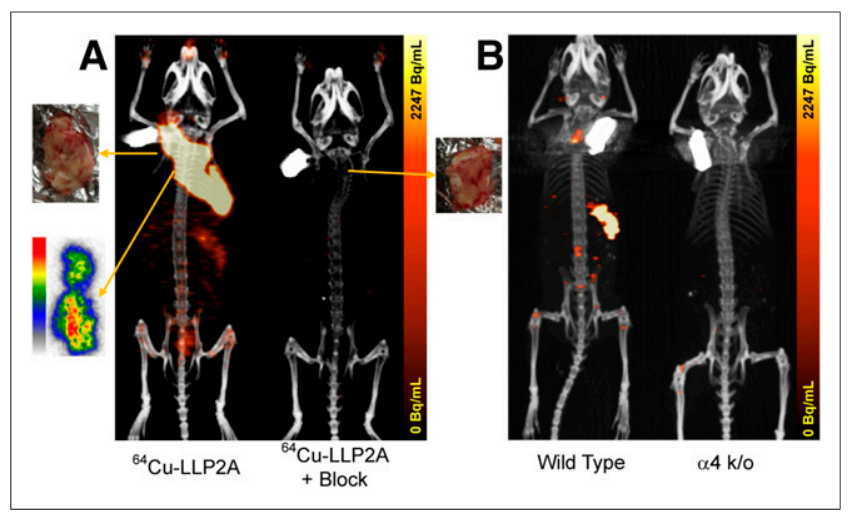

FIGURE 1. (A) Small-animal PET image of KaLwRij mouse bearing subcutaneous 5TGM1 tumor in nape of neck (left). PET/CT image was acquired at $24 \mathrm{~h}$ after injection of $0.48 \mathrm{MBq}$ of ${ }^{64} \mathrm{Cu}$-LLP2A. Also shown is autoradiographic $50-\mu \mathrm{m}$ coronal slice from center of frozen tumor imaged at $24 \mathrm{~h}$ after injection of $0.48 \mathrm{MBq}$ of ${ }^{64} \mathrm{Cu}$-LLP2A. In vivo uptake was blocked in presence of 200-fold excess of LLP2A (right). (B) Non-tumor-bearing wild-type KaLwRij mouse and an $\mathrm{a}_{4}$ knock-out (k/o) B6 mouse.

femur, tibia, pelvis, spinal cord, and spleen (Supplemental Fig. 4). KaLwRij mice injected intravenously with VLA-4-positive 5TGM1GFP MM cells were imaged over 3 weeks, demonstrating selective uptake of ${ }^{64} \mathrm{Cu}-\mathrm{LLP} 2 \mathrm{~A}$ in the expected lesions in the medullar cavity (pelvic bone and femur) and spleen. A representative set of maximumintensity-projection coronal and sagittal PET images of a mouse selected from a series of 4 mice after metastatic MM development, from before tumor inoculation (week 0) and at weeks 1, 2, and 3 after intravenous tumor inoculation, is shown in Figure 2A. Lesions were visualized in the femur-tibia knee joints and spleen as early as week 1. The SUV $\mathrm{Smax}_{\text {max }}$ and number of focal lesions in the medullar cavity further increased at weeks 2 and 3. The sagittal image of the mouse clearly shows several small lesions spread throughout the spine and the pelvic bone. The organ-to-muscle ratio of the

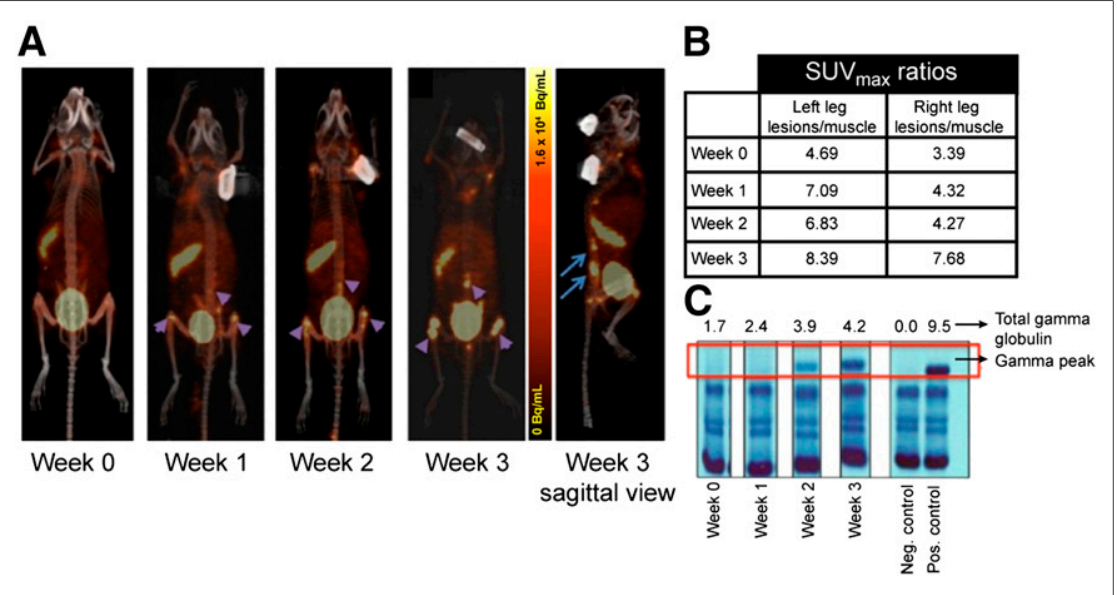

FIGURE 2. Small-animal PET/CT imaging using ${ }^{64} \mathrm{Cu}$-LLP2A in intravenous mouse model of 5TGM1 murine myeloma. (A) Representative images of KaLwRij mouse serially imaged over 3 weeks after tumor inoculation to monitor systemic medullar and extraosseous myeloma progression. Images were acquired at $4 \mathrm{~h}$ after injection of ${ }^{64} \mathrm{Cu}$-LLP2A ( $\left.\sim 0.74 \mathrm{MBq}\right)$. Sagittal view shows

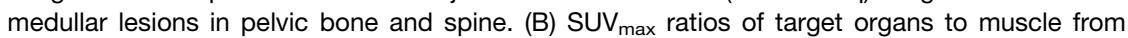
representative mouse as disease progressed. (C) Gel picture of serum protein electrophoresis of mouse shown in A starting at week 0 (before injection) till week 3 after injection. Numbers on top of gel represent total $\mathrm{y}$-globulin values in $\mathrm{g} / \mathrm{dL}$. prominent lesions in the left and right legs is presented in Figure 2B. The correlating serum protein electrophoresis gels with $\gamma$-peaks and values for the mice are shown in Figure 2C. The entire cohort of mice demonstrated a similar trend, and the leg bone SUVs averaged from $1.06 \pm 0.28$ at week 1 after tumor injection to $1.66 \pm 0.57$ at week 3 ( $n=4$ mice, 2 legs per mouse). Dynamic PET imaging (0-60 min) with ${ }^{64} \mathrm{Cu}$-LLP2A and ${ }^{18} \mathrm{~F}$-FDG at week 3 of tumor inoculation demonstrated comparable $\mathrm{SUV}_{\max }$ uptake in the prominent lesions in the femur-tibia proximity (Fig. 3).

\section{In Vitro Evaluations: Binding Specificity of LLP2A-Cy5 to Murine MM 5TGM1-GFP and Host Inflammatory Cells}

The VLA-4-positive 5TGM1-GFP murine myeloma cells were treated with LLP2A-Cy5, LLP2A-Cy5 mixed with unlabeled excess LLP2A, scrambled LLP2A-Cy5, or LLP2A-Cy5 mixed with firategrast, small-molecule $\alpha-4$ antagonist (Supplemental Fig. 5A) (15). In the VLA-4-positive 5TGM1-GFP cells, a significant percentage of the cells bound LLP2A-Cy5 (98.6\%) (14). The cell binding was competitively inhibited in the presence of cold competitor (LLP2A) or firategrast, coincubated at similar concentrations $(P<0.05)$. Scrambled LLP2A-Cy5 analog showed negligible binding to 5TGM1-GFP cells at the same concentration $(P<0.05)$. These data further demonstrate the specificity of LLP2A binding for activated/overexpressed VLA-4. Additionally, the competitive blocking effectiveness of LLP2A-Cy5 for ${ }^{64} \mathrm{Cu}-\mathrm{LLP} 2 \mathrm{~A}$ binding to VLA-4 on murine MM 5TGM1-GFP cells was statistically locking; $P<0.05$ ) (Supplemental Fig. 5B).

Binding of LLP2A-Cy5 to human MM cell lines, H929, RPMI8226, and OPM2 was also evaluated (14). Divalent cations such as $\mathrm{Mn}^{2+}$ induce integrin activation and are expected to increase the binding of LLP2A analogs to activated VLA-4 on myeloma cells $(17,18)$. To demonstrate this effect, cells were incubated in $\mathrm{Mn}^{2+}$-enriched medium, which significantly enhanced the binding of LLP2A-Cy 5 in the $\alpha_{4}$-positive cell lines compared with the normal medium (Supplemental Fig. 6). The cell binding was blocked in the presence of BIO5192, a selective and potent inhibitor of VLA-4.

We next evaluated the binding of LLP2A-Cy5 to different cell populations of bone marrow and splenic cells extracted from the syngeneic 5TGM1-GFP MM mouse model ( $n=$ 3 for all groups) using flow cytometry. Reference values for the flow cytometry analysis were obtained from non-tumorbearing mice ( $n=3$ for all groups). Flow cytometric analysis was performed on the bone marrow and splenic cells to evaluate binding of LLP2A-Cy5 to B cells (B220/ CD45R), T cells (CD4), and myeloid cells (Mac1 and Gr1) (Fig. 4). Specific binding of LLP2A-Cy5 was observed as expected to the VLA-4-expressing 5TGM1-GFP cells, which are also positive for the plasma $\mathrm{B}$ cell marker (B220). Interestingly, although LLP2A-Cy5 binding was negligible in B cells, T cells, and myeloid cells of tumor-naïve mice, these populations showed noticeable ectopic LLP2A-Cy5 binding cells from tumor-bearing mice (Supplemental Figs. 7 and 8 ). The background binding in the 


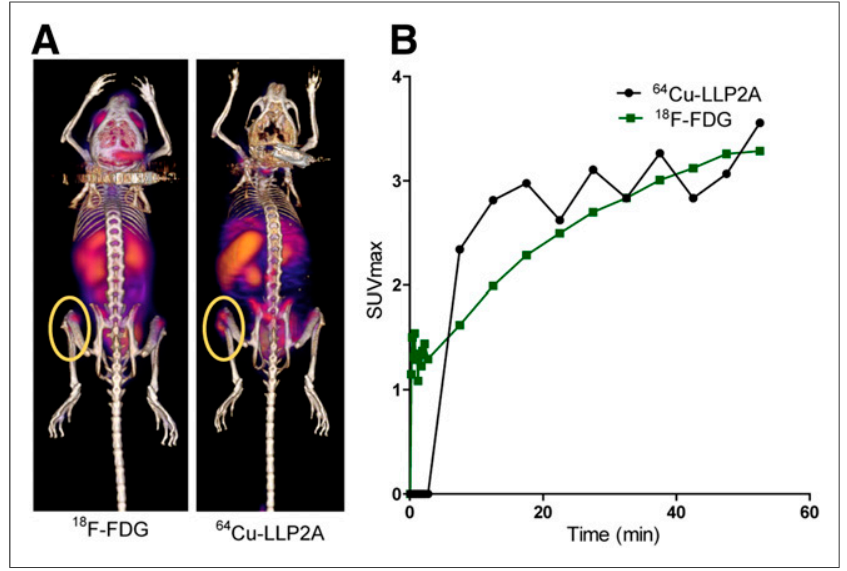

FIGURE 3. (A) Small-animal PET image at $60 \mathrm{~min}$ after injection of $10.9 \mathrm{MBq}$ of ${ }^{18} \mathrm{~F}-\mathrm{FDG}$ (left) and $0.74 \mathrm{MBq}$ of ${ }^{64} \mathrm{Cu}$-LLP2A (right). (B) Timeactivity curves derived from region of interest drawn on most prominent lesion in leg (0- to 60-min dynamic PET). Same mouse was imaged a day apart.

tumor-free immune-competent syngeneic KaLwRij mice was to CD4-positive T cells in the spleen and bone marrow, which express activated VLA-4.

\section{Human Absorbed Dose Estimates Extrapolated from Mouse Biodistribution Data}

Normal mouse biodistribution data with ${ }^{64} \mathrm{Cu}$-LLP2A were acquired at different time points (Supplemental Table 1). A substantial amount of radioactivity was observed in the urinary bladder, indi-

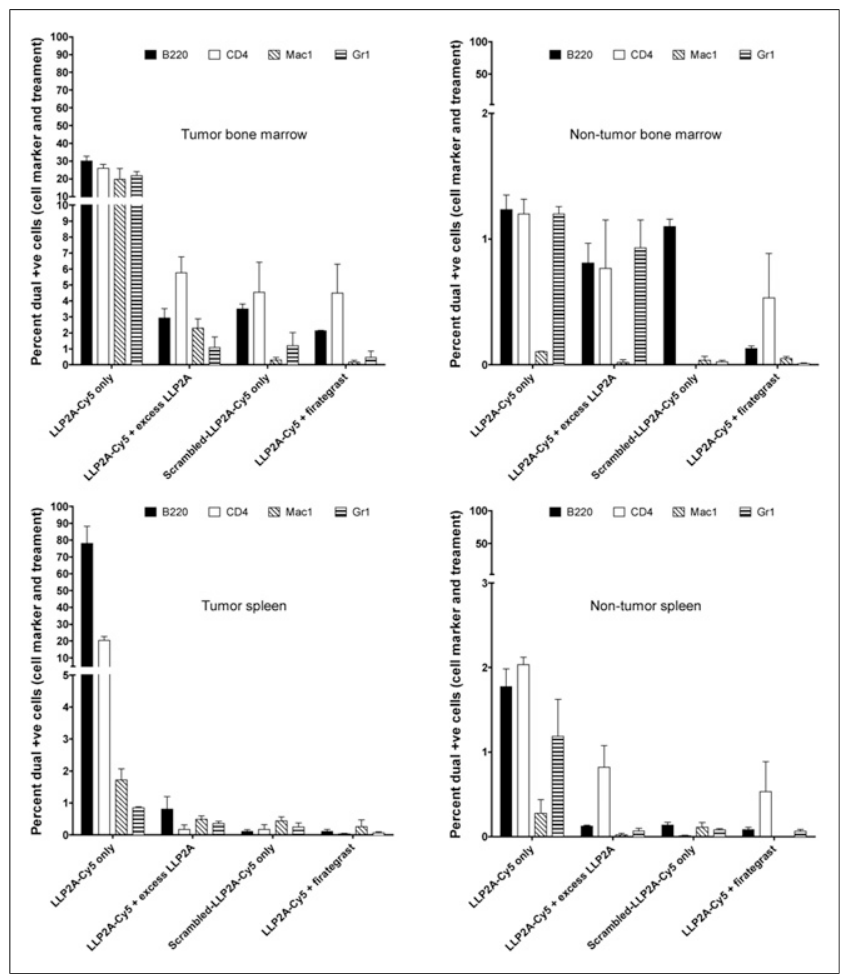

FIGURE 4. Fluorescence-activated cell sorting data of bone marrow and splenic cells in KaLwRij mice bearing intravenous 5TGM1-GFP tumors compared with age-matched KaLwRij mice ( $n=3$ for all groups). In tumor-bearing mice, more than $80 \%$ of B220-positive cells were 5TGM1GFP plasma tumor cells in both spleen and bone marrow. +ve = positive. cating urinary excretion as the major route of clearance starting at early time points. The cumulative percentage injected activity in urine collected in the metabolic cages along with the bladder filling fit $\left(\mathrm{A}_{0}=83 \%\right.$, and $\lambda_{\text {bio }}=0.91 / \mathrm{h}$, respectively) are shown in Figure $5 \mathrm{~A}$. These parameters were entered in the OLINDA bladder model using the 1-h void interval and resulted in a bladder content residence time of $0.45 \mathrm{~h}$. Because the area under the curve of the urine data correspond to a residence time of $14.46 \mathrm{~h}$, the urine excreted under the 1-h void assumption would correspond to $14.01 \mathrm{~h}$, which is expressed as residence time. The radioactivity also quickly cleared from the blood (Fig. 5B). The integrated organ residence times at 2-h void obtained from the mouse biodistribution data, scaled to human organ weight (Supplemental Table 2), were used to determine estimated human radiation doses (Table 1). The human radiation doses represent the normal dose per unit injected activity delivered to the normal organs in the standard-sized adult human male. On the basis of these data, the urinary bladder wall and spleen are the primary and secondary critical organs, respectively (19). The radioactivity cleared from the blood quickly with the time distribution fitted with a dualexponential function with a fast clearance component (6-min halflife) and a slower component with a 68-min half-life (Fig. 5B). On the basis of the scenario of a 1-h bladder-voiding interval, the bladder dose is $0.094 \mathrm{mGy} / \mathrm{MBq}$, whereas the red marrow dose was relatively low at $0.005 \mathrm{mGy} / \mathrm{MBq}$. The effective dose is $0.017 \mathrm{mSv} / \mathrm{MBq}$.

\section{LLP2A-Cy5 Staining of Primary Human Tissues}

To investigate whether LLP2A can be used to follow VLA-4 activation in human MM tissue, we stained 10 formalin-fixed paraffinembedded bone marrow samples from deidentified deceased MM patients with LLP2A-Cy5. Seven of the samples displayed a high proportion of cells intensely labeled with LLP2A-Cy5. In these

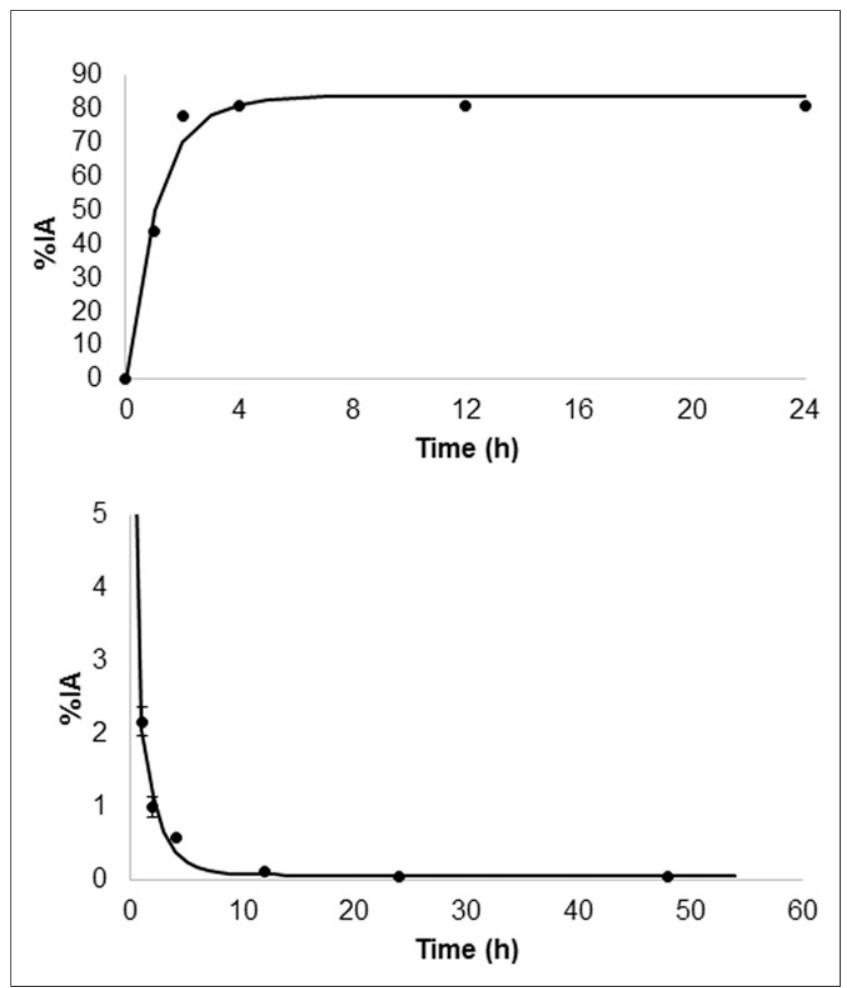

FIGURE 5. (Top) Time-activity curve for ${ }^{64} \mathrm{Cu}$-LLP2A (urine excretion). (Bottom) Time-activity curve for ${ }^{64} \mathrm{Cu}$-LLP2A (blood clearance). $\% \mathrm{IA}=$ percentage injected activity. 
TABLE 1

Extrapolated Human Radiation Dose Estimates for ${ }^{64} \mathrm{Cu}$-LLP2A

\begin{tabular}{ll}
\hline \multicolumn{1}{c}{ Organ } & Dose (mGy/MBq), 2-h void \\
\hline Adrenals & 0.005 \\
\hline Brain & 0.001 \\
\hline Breasts & 0.006 \\
\hline Gallbladder wall & 0.026 \\
\hline Large lower intestine wall & 0.012 \\
\hline Small intestine & 0.030 \\
\hline Stomach wall & 0.026 \\
\hline Upper large intestine wall & 0.010 \\
\hline Heart wall & 0.012 \\
\hline Kidneys & 0.013 \\
\hline Liver & 0.007 \\
\hline Lungs & 0.008 \\
\hline Muscle & 0.004 \\
\hline Ovaries & 0.009 \\
\hline Pancreas & 0.019 \\
\hline Red marrow & 0.021 \\
\hline Osteogenic cells & 0.047 \\
\hline Skin & 0.005 \\
\hline Spleen & 0.032 \\
\hline Testes & 0.004 \\
\hline Thymus & 0.018 \\
\hline Urinary bladder wall & 0.004 \\
\hline Eterus & 0.741 \\
\hline Effective dose equivalent & 0.012 \\
\hline
\end{tabular}

cells, LLP2A exhibited punctate staining around the cell periphery, consistent with it labeling VLA-4 (Fig. 6). The 3 other tissue samples displayed few LLP2A-positive cells.

\section{DISCUSSION}

VLA-4 is a transmembrane adhesion receptor expressed on normal plasma cells that is an important contributor to plasma cell interactions with the extracellular matrix and bone marrow stromal cells. MM is a hematologic malignancy characterized by the clonal proliferation of plasma cells within the bone marrow. Multimodal imaging (PET with CT or MRI) might provide additional critical information such as predicting high-risk fracture sites, visualizing oligosecretory MM tumors, and assessing treatment response (20). Several pathways activated by plasma cell interactions with different bone marrow cell types have been shown to affect the behavior of the malignant myeloma plasma cells $(21,22)$. A variety of cell types including macrophages, osteoclasts, and T lymphocytes produce cytokines and other factors constituting a microenvironment that is conducive for the growth and development of myeloma plasma cells $(23,24)$. Myeloma cells specifically adhere to the bone marrow extracellular matrix proteins fibronectin and vascular cell adhesion molecule-1, through the integrin VLA-4, which contributes to localization of tumor cells in the bone marrow (25). A body of evidence also supports the fact that bone marrow stromal cells are indispensable for the differentiation, growth, and homing of the myeloma cells in the bone marrow (26-29). Here, we sought to evaluate the binding specificity of VLA-4targeting high-affinity peptidomimetic LLP2A ligand-based optical and nuclear bioconjugates for cell populations that express activated VLA-4 in normal and intravenous MM-bearing mice.

The small-animal ${ }^{64} \mathrm{Cu}-\mathrm{LLP} 2 \mathrm{~A}$ PET/CT images clearly showed myeloma lesions in the osseous and extraosseous tissues in the disseminated and subcutaneous murine myeloma models. The radiopharmaceutical uptake was significantly reduced in $\alpha_{4}$ knock-out mice and in the presence of competitor ligands in tumor-bearing wild-type mice, demonstrating in vivo specificity. The 5TGM1-GFP-transplanted C57BL6/KaLwRij mouse model is widely used to study murine MM pathogenesis. It recapitulates distinct features of human MM including monoclonal paraprotein as well as lytic bone lesions (30). Using this mouse model, we characterized the binding specificity of LLP2A to different cell subsets in the spleen and bone marrow of the C57BL6/KaLwRij mice and defined differences in the binding of LLP2A to a subset of profiles between 5TGM1-GFP tumor- and non-tumor-bearing C57BL6/KaLwRij mice.

Activated VLA-4 expression has been reported on various cell populations such as B cells and $\mathrm{T}$ cells and is also known to be coexpressed with cell markers such as Gr1 and Mac1. Binding of LLP2A-Cy5 was evaluated in the B cells, T cells, myeloid cells, and macrophage and monocytes extracted from the mouse spleen and bone marrow. In non-tumor-bearing mice, we found that CD4 cells had the highest staining with LLP2A-Cy5, which explained the background signal consistently observed in the spleen. Specific binding of LLP2A-Cy5 to tumor cells was observed in the 5TGM1-GFP tumorbearing KaLwRij mice. Interestingly, in the bone marrow of tumorbearing mice, the staining of cell types such as granulocytes, monocyte/macrophages, and $\mathrm{T}$ cells also increased compared with

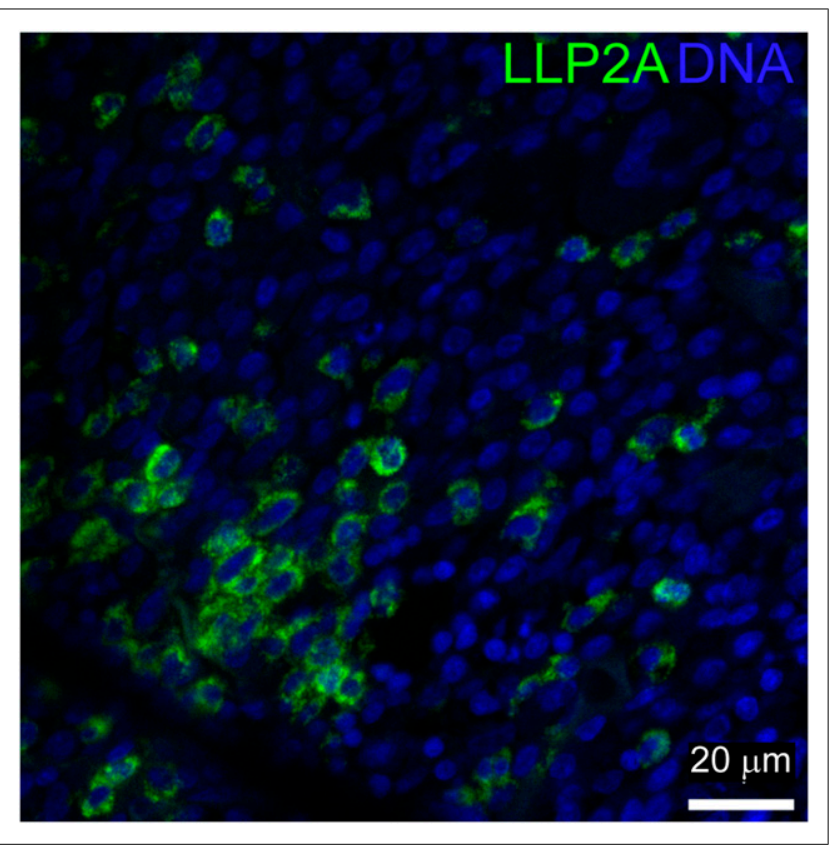

FIGURE 6. Representative image of myeloma patient's sample with high levels of LLP2A-Cy5 staining. LLP2A staining is shown in green and 4',6-diamidino-2-phenylindole (DAPI) labeling of nuclei in blue. 
normal mice. These data suggest that the presence of tumor cells in the bone marrow induces an inflammatory microenvironment characterized by increased levels of VLA-4 positivity on multiple cell types. The overall binding to stromal cells was significantly lower in the tumor-naïve mice. This finding supports a direct effect of myeloma on VLA-4 activation and expression in host nonmalignant bone marrow and splenic hematopoietic microenvironmental elements.

Animal biodistribution and radiation dosimetry estimates indicated a fast blood clearance and high urinary excretion, leading to favorable radiation doses for imaging with an effective dose of $0.017 \mathrm{mSv} / \mathrm{MBq}$. The high excretion in the urine led to the urinary bladder wall being the dose-limiting organ at $0.200 \mathrm{mGy} / \mathrm{MBq}$. This value indicated a maximum injection of $532 \mathrm{MBq}$ of ${ }^{64} \mathrm{Cu}-\mathrm{LLP} 2 \mathrm{~A}$, assuming a maximum permissible dose of $50 \mathrm{mGy}$ to the bladder wall. In addition, the absorbed dose to the bone marrow in normal mice was low, making the ligand amenable to VLA-4-targeted bone marrow therapies. On the basis of our in vitro and in vivo data, the dosimetry in tumor-bearing mice is expected to show enhanced uptake in the spleen and other immune organs, including the bone marrow. Preliminary staining of the human tissues from deceased myeloma patients showed a heterogeneous population of VLA-4 positive and -negative cells, a likely projection of patient-to-patient variability seen in myeloma spectrum diseases. Additional studies with primary MM samples are warranted to evaluate the relationship between LLP2A staining and clinical parameters.

\section{CONCLUSION}

Favorable binding characteristics, animal biodistribution, and dosimetry and reproducible production of high-specific-activity product make ${ }^{64} \mathrm{Cu}-\mathrm{LLP} 2 \mathrm{~A}$ a promising candidate for imaging activated VLA-4 in humans.

\section{DISCLOSURE}

The costs of publication of this article were defrayed in part by the payment of page charges. Therefore, and solely to indicate this fact, this article is hereby marked "advertisement" in accordance with 18 USC section 1734 . This research was supported by the NIH NCI R01CA176221, NIH NCI U54CA199092, and NIH NCI 1R21CA180211. No other potential conflict of interest relevant to this article was reported.

\section{ACKNOWLEDGMENTS}

We thank Drs. Katherine N. Weilbaecher and Kooresh I. Shoghi for suggestions and valuable criticisms. Dr. Jinbin Xu helped with the autoradiography studies. We are grateful to Dr. Ravi Vij and the Division of Oncology for supplying deidentified FFPE human tissues. We thank the Musculoskeletal Research Center for histology (NIH P30 AR057235), the MIR preclinical PET/CT and the cyclotron facility, the P50 small-animal imaging core for optical imaging (CA94056).

\section{REFERENCES}

1. Anderson KC, Alsina M, Bensinger W, et al. Multiple myeloma, version 1.2013. J Natl Compr Canc Netw. 2013;11:11-17.

2. Kuehl WM, Bergsagel PL. Molecular pathogenesis of multiple myeloma and its premalignant precursor. J Clin Invest. 2012;122:3456-3463.

3. Rajkumar SV, Dimopoulos MA, Palumbo A, et al. International myeloma working group updated criteria for the diagnosis of multiple myeloma. Lancet Oncol. 2014;15:e538-e548.
4. Wang TF, Ahluwalia R, Fiala MA, et al. The characteristics and outcomes of patients with multiple myeloma dual refractory or intolerant to bortezomib and lenalidomide in the era of carfilzomib and pomalidomide. Leuk Lymphoma. 2014;55:337-341.

5. van Lammeren-Venema D, Regelink JC, Riphagen II, Zweegman S, Hoekstra OS, Zijlstra JM. ${ }^{18}$ F-fluoro-deoxyglucose positron emission tomography in assessment of myeloma-related bone disease: a systematic review. Cancer. 2012;118:1971-1981.

6. Schlesinger M, Bendas G. Contribution of very late antigen-4 (VLA-4) integrin to cancer progression and metastasis. Cancer Metastasis Rev. 2015;34:575-591.

7. Sanz-Rodríguez F, Teixido J. VLA-4-dependent myeloma cell adhesion. Leuk Lymphoma. 2001;41:239-245.

8. Sanz-Rodríguez F, Hidalgo A, Teixido J. Chemokine stromal cell-derived factor1alpha modulates VLA-4 integrin-mediated multiple myeloma cell adhesion to CS-1/fibronectin and VCAM-1. Blood. 2001;97:346-351.

9. Nishinarita S, Shimada H, Ito T, et al. Expression of beta 1 integrins (very late antigens-4 and -5) on myeloma cells and clinical correlates in patients with multiple myeloma. J Int Med Res. 1998;26:37-42.

10. Peng L, Liu R, Andrei M, Xiao W, Lam KS. In vivo optical imaging of human lymphoma xenograft using a library-derived peptidomimetic against alpha4beta1 integrin. Mol Cancer Ther. 2008;7:432-437.

11. Peng L, Liu R, Marik J, Wang X, Takada Y, Lam KS. Combinatorial chemistry identifies high-affinity peptidomimetics against alpha4betal integrin for in vivo tumor imaging. Nat Chem Biol. 2006;2:381-389.

12. Soodgupta $D$, Hurchla MA, Jiang $M$, et al. Very late antigen-4 ( $\alpha_{4} \beta_{1}$ integrin) targeted PET imaging of multiple myeloma. PLoS One. 2013;8:e55841.

13. Beaino W, Anderson CJ. PET imaging of very late antigen-4 in melanoma: comparison of ${ }^{68} \mathrm{Ga}$ - and ${ }^{64} \mathrm{Cu}$-labeled NODAGA and CB-TE1A1P-LLP2A conjugates. J Nucl Med. 2014;55:1856-1863.

14. Soodgupta D, Pan D, Cui G, et al. Small molecule MYC inhibitor conjugated to integrin-targeted nanoparticles extends survival in a mouse model of disseminated multiple myeloma. Mol Cancer Ther. 2015;14:1286-1294.

15. Rettig MP, Ansstas G, DiPersio JF. Mobilization of hematopoietic stem and progenitor cells using inhibitors of CXCR4 and VLA-4. Leukemia. 2012;26:34-53.

16. Priestley GV, Ulyanova T, Papayannopoulou T. Sustained alterations in biodistribution of stem/progenitor cells in Tie2Cre + alpha4(f/f) mice are hematopoietic cell autonomous. Blood. 2007;109:109-111.

17. Masumoto A, Hemler ME. Multiple activation states of VLA-4. Mechanistic differences between adhesion to CS1/fibronectin and to vascular cell adhesion molecule-1. J Biol Chem. 1993;268:228-234.

18. Dormond O, Ponsonnet L, Hasmim M, Foletti A, Ruegg C. Manganese-induced integrin affinity maturation promotes recruitment of alpha $\mathrm{V}$ beta 3 integrin to focal adhesions in endothelial cells: evidence for a role of phosphatidylinositol 3-kinase and Src. Thromb Haemost. 2004;92:151-161.

19. Laforest R, Dehdashti F, Lewis JS, Schwarz SW. Dosimetry of $60 / 61 / 62 /{ }^{64} \mathrm{Cu}$-ATSM: a hypoxia imaging agent for PET. Eur J Nucl Med Mol Imaging. 2005;32:764-770.

20. Mulligan M, Chirindel A, Karchevsky M. Characterizing and predicting pathologic spine fractures in myeloma patients with FDG PET/CT and MR imaging. Cancer Invest. 2011;29:370-376.

21. Burger JA, Ghia P, Rosenwald A, Caligaris-Cappio F. The microenvironment in mature B-cell malignancies: a target for new treatment strategies. Blood. 2009;114:33673375 .

22. Zhuang J, Zhang J, Lwin ST, et al. Osteoclasts in multiple myeloma are derived from Gr-1+CD11b+myeloid-derived suppressor cells. PLoS One. 2012;7:e48871.

23. Fowler JA, Mundy GR, Lwin ST, Edwards CM. Bone marrow stromal cells create a permissive microenvironment for myeloma development: a new stromal role for Wnt inhibitor Dkk1. Cancer Res. 2012;72:2183-2189.

24. Teoh G, Urashima M, Greenfield EA, et al. The 86-kD subunit of Ku autoantigen mediates homotypic and heterotypic adhesion of multiple myeloma cells. J Clin Invest. 1998;101:1379-1388.

25. Uchiyama H, Barut BA, Chauhan D, Cannistra SA, Anderson KC. Characterization of adhesion molecules on human myeloma cell lines. Blood. 1992;80:2306-2314.

26. Hallek M, Bergsagel PL, Anderson KC. Multiple myeloma: increasing evidence for a multistep transformation process. Blood. 1998;91:3-21.

27. Uchiyama H, Barut BA, Mohrbacher AF, Chauhan D, Anderson KC. Adhesion of human myeloma-derived cell lines to bone marrow stromal cells stimulates interleukin-6 secretion. Blood. 1993;82:3712-3720.

28. Masellis-Smith A, Belch AR, Mant MJ, Pilarski LM. Adhesion of multiple myeloma peripheral blood B cells to bone marrow fibroblasts: a requirement for CD44 and alpha4beta7. Cancer Res. 1997;57:930-936.

29. Urashima M, Chen BP, Chen S, et al. The development of a model for the homing of multiple myeloma cells to human bone marrow. Blood. 1997;90:754-765.

30. Vanderkerken K, De Raeve H, Goes E, et al. Organ involvement and phenotypic adhesion profile of 5T2 and 5T33 myeloma cells in the C57BL/KaLwRij mouse. Br J Cancer: 1997;76:451-460. 